Henry Gardner • Gabriele Manduchi

\title{
Design Patterns \\ for e-Science
}

With 60 Figures

Springer 


\section{Contents}

\section{Part I Construction of a Waveform Browser}

1 e-Science and EScope $\ldots \ldots \ldots \ldots \ldots \ldots \ldots \ldots \ldots \ldots \ldots \ldots$

1.1 What is this Thing Called "e-Science"?.............. 3

1.2 Computers in Physics . . . . . . . . . . . . . . . . . 5

1.3 Computers in Fusion Energy Research ............... 5

1.4 Programming Languages and Operating Systems .......... 8

1.5 Our Target Data Server: MDSplus .................. 9

1.6 Two Sample Datasets . . . . . . . . . . . . . . . . . . . . . 10

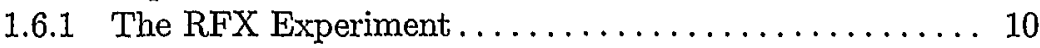

1.6 .2 The $\mathrm{H}-1 \mathrm{NF}$ Heliac $\ldots \ldots \ldots \ldots \ldots \ldots \ldots \ldots \ldots \ldots \ldots \ldots$

$1.7 \mathrm{jScope}$ and EScope $\ldots \ldots \ldots \ldots \ldots \ldots \ldots \ldots \ldots \ldots$

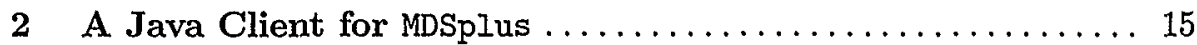

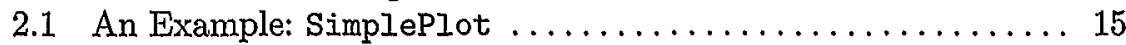

2.2 Java IO . . . . . . . . . . . . . . . . . . . . 17

2.2.1 A Remark on Exceptions ................. 17

2.2.2 Character-Based Text Streams ............... 18

2.2 .3 Input from the Keyboard . . . . . . . . . . . . 19

2.2 .4 Writing Text Output .................. 19

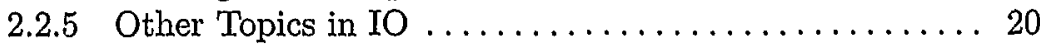

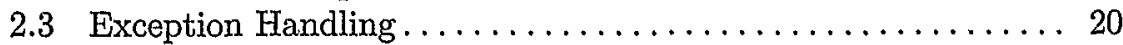

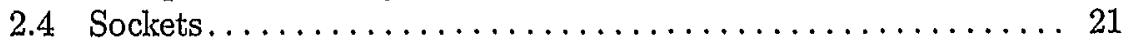

2.4.1 A Socket Example: Requesting Data from a Server . . . 22

2.5 Introduction to Threads . . . . . . . . . . . . . . . 25

2.5.1 Threaded Plot Server ................... 26

2.6 A Java API for MDSplus . . . . . . . . . . . . . . . 28

2.7 The Data Organization of MDSplus ............... 30

2.7.1 The mdsip Protocol for Remote Data Access . . . . . . . . 32

2.7 .2 Operation of MDSMessage ................ 34

2.7.3 Operation of MDSNetworkSource ............ 35 
2.8 PreEScope0: A Program to Connect to MDSPlus . . . . . . . . 35

2.9 Programming Exercises . . . . . . . . . . . . . . . 36

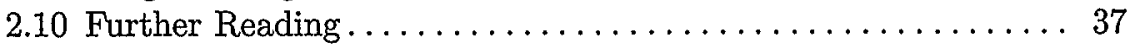

3 Graphical User Interfaces Using Swing . . . . . . . . . 39

3.1 Simple GUI Programming . . . . . . . . . . . . . . 39

3.1 .1 A Blank Frame ..................... 39

3.1.2 Laying-out Components in a JPanel . . . . . . . . . . . 41

3.1.3 A Bizarre Component Frame ............... 43

3.2 A Note on Programming Style . . . . . . . . . . . . . 45

3.3 A Look Inside Plotter . . . . . . . . . . . . . . . . . . . . . . . . . 46

3.4 Action Listeners in Swing $\ldots \ldots \ldots \ldots \ldots \ldots \ldots \ldots \ldots \ldots \ldots \ldots$

3.5 Swing Miscellany . . . . . . . . . . . . . . . . . 50

3.5.1 Text Fields and the Model-View-Controller Design

Pattern .......................... 52

3.6 PreEScope1: A Simple GUI for PreEScope0 . . . . . . . . . . . . 54

3.6.1 Using JOptionPane to Request Information . . . . . . . . 54

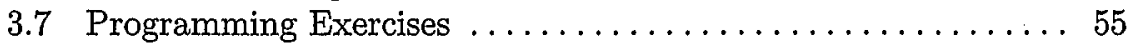

3.8 Further Reading ....................... 56

4 Waveform Graphics...................... 57

4.1 Java2D Graphics . . . . . . . . . . . . . . . . 57

4.2 A Second Look at Plotter .................. 58

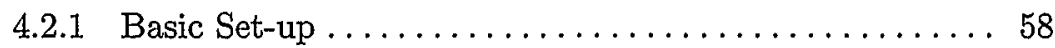

4.2 .2 Setting Colors and Strokes............... 59

4.2 .3 Transform Data and Plot the Line ............... 60

4.3 A Fancier Plot ......................... 62

4.3 .1 Fonts .......................... 63

4.3.2 Calculating Border Dimensions . . . . . . . . . . . . 64

4.3.3 Drawing Titles and Axis Labels . . . . . . . . . . . . 66

4.3 .4 Filtering Data . . . . . . . . . . . . . . . 66

4.3.5 Overall Structure of paintComponent for an Adorned Graph ....................... 68

4.4 Axis Calculations: Tick Positions, Tick Values and Scientific

Notation . . . . . . . . . . . . . . . . . . . . . . . . . 69

4.5 PreEScope2: Nicer Graphs from MDSplus . . . . . . . . . . . . 71

4.6 Programming Exercises . . . . . . . . . . . . . . . . 71

4.6.1 Programming Exercises . . . . . . . . . . . . . 71

4.7 Further Reading...................... 72

5 Interactive Graphics Using Mouse Events. . . . . . . . . . . 73

5.1 Mouse Interfaces and Events . . . . . . . . . . . . . . . 74

5.1.1 The MouseListener Interface ............... 74

5.1 .2 The MouseMotionListener Interface . . . . . . . . . . . 74

5.1 .3 The MouseEvent Class ..................... 75 
5.2 PreEscope3: The Graph Point Diagnostic ............. . 75

5.2.1 OpenGL Hardware Acceleration . . . . . . . . . . . . . 77

5.3 Programming Exercises . . . . . . . . . . . . . . . 78

5.4 Further Reading ....................... 78

6 Navigating the Database . . . . . . . . . . . . . . 79

6.1 Custom-Built Linked Lists . . . . . . . . . . . . . . . 80

6.2 Lists in Swing . . . . . . . . .

6.2.1 Using the DefaultListModel Class . . . . . . . . . . . 84

6.2 .2 Using the ListModel Interface . . . . . . . . . . . . . . . 84

6.2 .3 Rendering List Cell Values . . . . . . . . . . . 85

6.3 Trees . . . . . . . . . . . . . . . . . . . . . . . . . . 85

6.3 .1 Recursion $\ldots \ldots \ldots \ldots \ldots \ldots \ldots \ldots \ldots \ldots \ldots \ldots \ldots$

6.3.2 Using Recursion to Probe File Structures . . . . . . . . . 87

6.4 Trees in Swing . . . . . . . . . . . . . . . . . . 88

6.4.1 Tree Paths and Tree Selection Listeners . . . . . . . . . . 88

6.4 .2 Tree Cell Rendering . . . . . . . . . . . . . . . . . 89

6.5 MDSTree and MDSTreeNode . . . . . . . . . . . . . . . . 89

6.5.1 Reading the MDSPlus Experiment Hierarchy ....... 91

6.6 PreEScope4: A Waveform Browser for MDSplus ............. 93

6.6.1 Issues to Consider for PreEScope $\ldots \ldots \ldots \ldots \ldots \ldots \ldots$

6.7 Further Reading...................... 97

\section{Part II Refactoring EScope with Design Patterns}

7 Object-Oriented Analysis and Design .............. 101

7.1 Phases of Software Development. . . . . . . . . . . . . . 101

7.2 UML and Design Patterns . . . . . . . . . . . . . . . . . 104

7.3 Design Patterns: Our Approach . . . . . . . . . . . . . . . 105

7.4 A Diagrammatic Notation: "sUML" ................. 105

7.4 .1 Associations . . . . . . . . . . . . . . . . . . 106

7.4 .2 Association Multiplicities . . . . . . . . . . . . . 106

7.4 .3 Association Labels. . . . . . . . . . . . . . . . . 108

7.4 .4 Reflexive Associations. . . . . . . . . . . . . . . . 108

7.4 .5 Ignore Aggregation! . . . . . . . . . . . . . . . 109

7.4 .6 Dependency . . . . . . . . . . . . . . . . . . 109

7.4 .7 Package Associations . . . . . . . . . . . . . . 110

7.4 .8 Inheritance and Implementation . . . . . . . . . 110

7.5 Summary of Our sUML Class Diagrams . . . . . . . . . . . 111

7.6 Further Reading............................ 112 


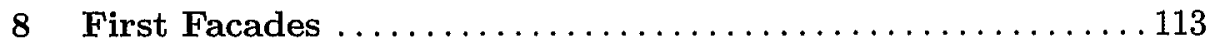

8.1 Facade ................................. 114

8.2 EScope0: A "Do Nothing" Code Refactoring Using Packages . . 114 8.2 .1 Using Makefiles .......................... 118

8.3 EScope1: First Implementation of the Facade Pattern . . . . . . 119

8.3.1 Place Facade Interfaces into a Shared Package ....... 119

8.3.2 Facade Interface for the GUI Domain ............ 120

8.3.3 Facade Interface for the Data Server Domain ........ 120

8.3.4 Facade Interface for the Graphics Domain .......... 121

8.3.5 Our Final Product . . . . . . . . . . . . . . . . . . . . 121

8.4 The Mediator Pattern........................ 123

8.5 More Notes on Facade and Mediator Patterns ........... 125

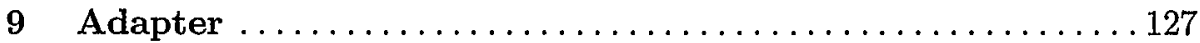

9.1 Object Adapter Pattern . . . . . . . . . . . . . . . . . . 127

9.2 Class Adapter Pattern ........................ 129

9.3 Are Object Adapters Better Than Class Adapters? . . ....... 130

9.4 EScope2: Sharing Graph Data and Graph Options Between

Domains ................................ 130

9.4.1 Passing Graph Options from the User Interface ....... 132

9.4 .2 An Articulated Facade ...................... 132

9.4 .3 Our Final Product ....................... 133

9.4.4 Data Server Domain ........................... 133

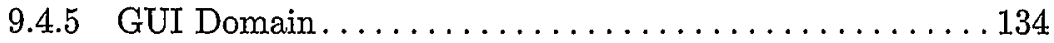

9.4.6 Graphics Domain.................... 136

10 The Template Pattern . . . . . . . . . . . . . . . . . . . 139

10.1 Pattern Description . . . . . . . . . . . . . . . . . . . 139

10.2 EScope3: Splitting up the Graphics Facade ............ 140

10.2.1 The GraphData and GraphMetrics Classes ..........143

10.2.2 Drawing Individual Graph Components ........... 143

10.2.3 The Template Pattern for DrawAxesTicks ........... 144

10.2.4 Our Final Product ........................ 144

11 Decorator ..................................... 147

11.1 Pattern Description......................... 147

11.2 EScope4: Adding Zoom and Grab Options Using the

Decorator Pattern ............................. 149

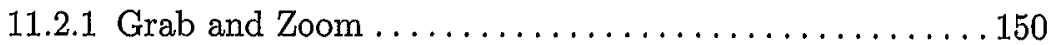

11.2.2 A Mediator Emerges ........................ 150

11.2.3 Our Final Product ....................... 152 
12 Patterns at Work: Multiple Waves . . . . . . . . . . . . . . 155

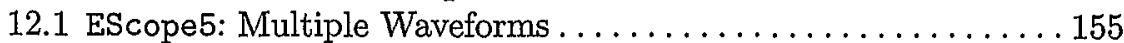

12.1 .1 Requirements . . . . . . . . . . . . . . . . . . 155

12.1.2 Interfaces and External Requirements . . . . . . . . . 156

12.1.3 Plotting an Array of Waveforms . . . . . . . . . . . . 157

12.1.4 Modifications to the GUI Domain . . . . . . . . . . . 158

12.1.5 Drawing the Cross-Hair . . . . . . . . . . . . . . 160

12.2 Our Final Product............................ 162

13 Patterns at Work: Multiple Graphs . . . . . . . . . . . 165

13.1 EScope6: Multiple Windows in EScope .............. 165

13.1.1 Designing for Multiple Windows . . . . . . . . . . . . . 167

13.1.2 The Flexible Grid Layout Manager . . . . . . . . . . . . . . 169

13.2 Image Buffering: A Useful Graphics Trick . . . . . . . . . . . . . . . 170

13.3 Our Final Product. . . . . . . . . . . . . . . . . . . 171

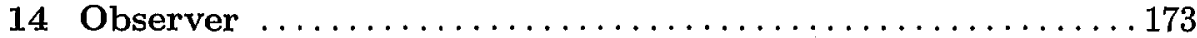

14.1 Pattern Description . . . . . . . . . . . . . . . 173

14.2 EScope7: Integrating Synchronized Interaction in Multiple

Windows ................................ 175

14.2.1 sharedObserverInterfaces Completes the

Articulated Facade . . . . . . . . . . . . . 175

14.2.2 Management of a Collection of Graphics Facades . . . . 177

14.2.3 The Graph Scale Interfaces . . . . . . . . . . . . . . . 179

14.2.4 Our Final Product ................. 180

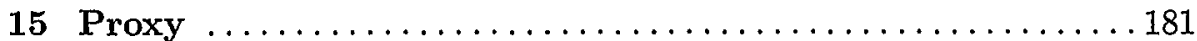

15.1 EScope8: Implementation of a Local Data Cache . . . . . . . . 182

15.1.1 The DataServerProxy Class . . . . . . . . . . . . . . 182

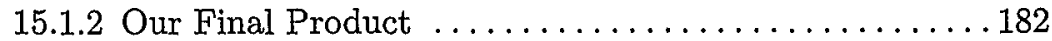

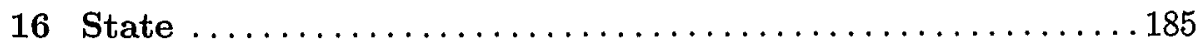

16.1 Pattern Description . . . . . . . . . . . . . . . . . 185

16.2 Escope9: A State Pattern for the DataServerFacade . . . . . . 186

16.2.1 Common Interface . . . . . . . . . . . . . . . 187

16.2.2 State Inner Classes . . . . . . . . . . . . . . . . . 187

16.2.3 Managing State Transitions . . . . . . . . . . . . 188

16.3 Our Final Product. . . . . . . . . . . . . . . . . . . . . . 189

17 Factory Patterns............................... 191

17.1 A Factory Tour . . . . . . . . . . . . . . . . . . . 191

17.1.1 Informal Factory Methods . . . . . . . . . . . . . . . 191

17.1.2 The Factory Method Pattern ................ 192

17.1.3 Abstract Factory . . . . . . . . . . . . . . . . . . 192

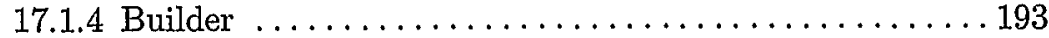


17.1.5 Prototype .............................. 193

17.1.6 Singleton ............................. 193

17.2 EScope10: Multiple Data Servers ................... 193

17.2.1 Requirements ........................... 194

17.2.2 Implementation ........................ 195

17.2.3 Example Properties File..................... 196

17.2.4 The ServerSelectDialog. ................ 197

17.2.5 The Factory Pattern in EScope10 . . . . . . . . . . 197

17.2.6 A Text Data Server.......................... 198

17.3 Our Final Product........................... 199

18 Chain of Responsibility ........................ 201

18.1 EScope11: Avoiding Explicit Connection to Data Servers .....201

18.1.1 An Example Properties File ...................201

18.1.2 Implementation ........................202

18.1.3 Our Final Product .......................202

19 Design Patterns and Threads .................... 203

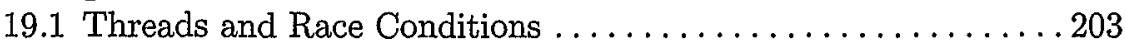

19.2 Synchronized Methods and wait()/notify() ..........206

19.3 Patterns for Concurrent Systems . . . . . . . . . . . . . . . 208

19.3.1 The Acceptor-Connector Pattern . . . . . . . . . . . . . . 209 209

19.3.2 The Asynchronous Method Pattern ............. 209

19.3.3 More Complete Implementations of AbstractNotifier 211

19.3.4 Other Classes in the Asynchronous Method Pattern . . . 213

19.3.5 Summary of the Asynchronous Method Pattern ....... 214

19.3.6 The Active Object Pattern ..................214

19.4 EScope12: A Progress Bar for Downloading Signals .........215

19.4.1 Using Threads with Swing ................... 216

19.5 Programming Exercises . . . . . . . . . . . . . . . . . . 217

19.6 Further Reading ............................. 217

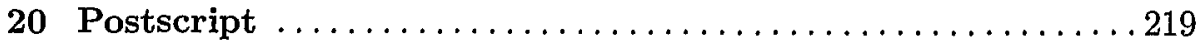

20.1 Design Patterns Then and Now ........................ 219

20.2 The e-Science "Software Stack" . . . . . . . . . . . . . . . 220

20.3 Server-Side EScope for DataGrids (with Raju Karia) . ........222

20.3.1 Metadata Indexing, Persistence and Provenance in WebScope ............................223

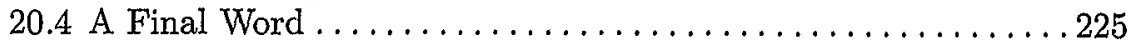

A Installing and Running Data Servers for EScope ........227

A.1 The MdsipSimulator Program ....................227

A.2 The Text Data Server .......................... 228

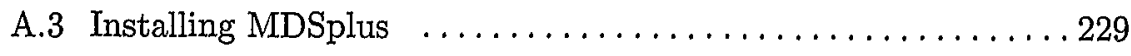


A.3.1 Installing on Microsoft Windows Using the Install Shield on the CD . .....................229

A.3.2 Installing on Windows from www.mdsplus .org . . . . . 229

A.3.3 Installing on Linux Using the Supplied RPM ........ 230

A.4 Running MDSplus with the Sample Data.............. 231

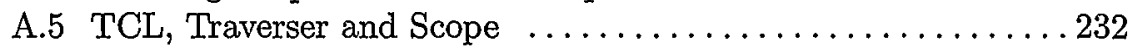

A.5.1 Creating a Simple Database Using TCL ........... 232

A.5.2 Examining a Database Using the Traverser ......... 234

A.5.3 Creating and Viewing Subtrees............... 235

A.5.4 Understanding Node Names ................ 236

A.5.5 Defining Signals and Viewing Them with jScope ..... 238

A.5.6 UNITS_OF() and DIM_OF() $\ldots \ldots \ldots \ldots \ldots \ldots \ldots \ldots \ldots \ldots \ldots$

B Listings of Introductory Examples ................. 243

B.1 BorderComponentFrame ........................243

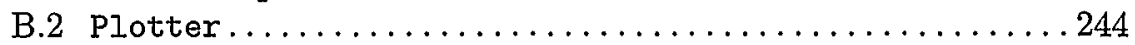

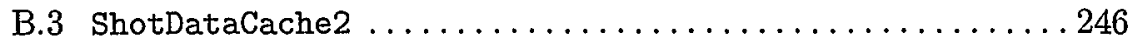

C Helper Classes for Accessing MDSplus from Java ......... 249

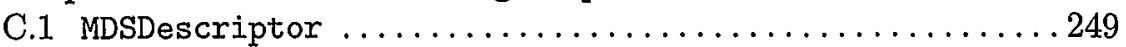

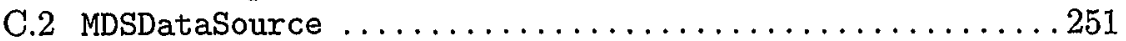

C.3 MDSNetworkSource ..........................252

C.4 MDSMessage ...............................256

D Listings for PreEScope Examples .................263

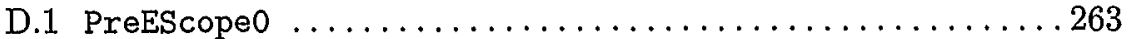

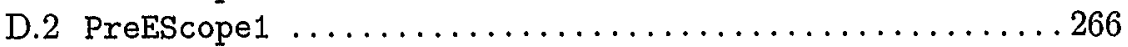

D.2.1 PreEScope1 Main Program ...................266

D.2.2 EScopeFrame Class .......................267

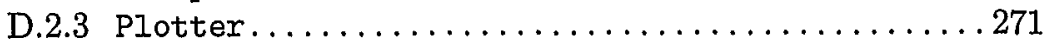

D.3 PreEScope2 ...............................271

D.4 MDSTree and MDSTreeNode ....................280

D.4.1 MDSTreeNode ...........................280

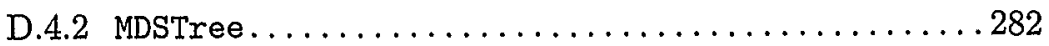

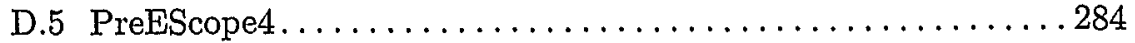

D.5.1 EScopeFrame ............................285

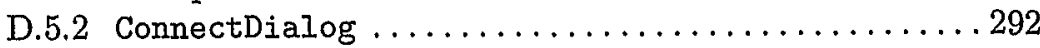

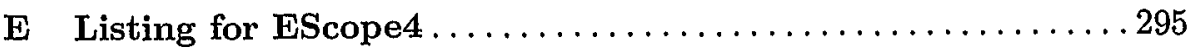

E.1 Package Structure . ........................... 295

E.2 Shared Data Interfaces . . . . . . . . . . . . . . . . . . 296

E.3 Shared Interfaces . . . . . . . . . . . . . . . . . . . . . 298

E.3.1 DataServerFacadeInterface..................298

E.3.2 GraphicsFacadeInterface .................... 299

E.3.3 GuiFacadeInterface ........................299 
E.3.4 AbstractGraphicsFacade ................... 299

E.4 The Data Server Package ......................... 300

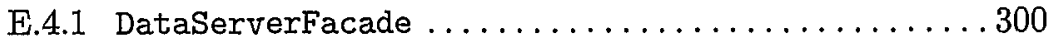

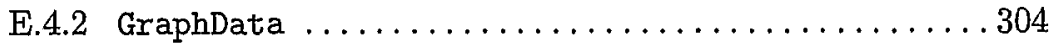

E.5 The Graphics Domain ......................... 305

E.5.1 The Decorator Classes ....................... 306

E.5.2 Adapter Classes ............................. 320

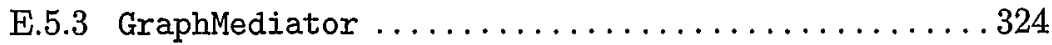

E.5.4 GraphicsFacade ............................ 330

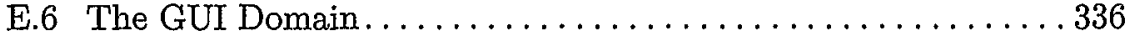

E.6.1 The Dialog Classes ........................... 336

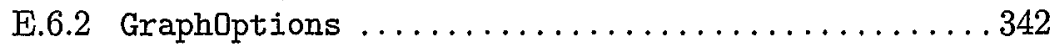

E.6.3 GuiFacade ................................. 344

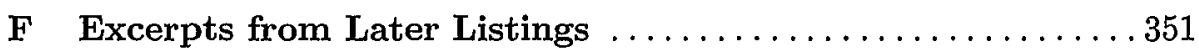

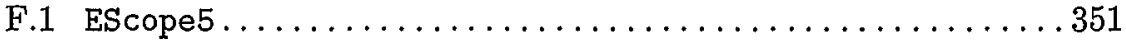

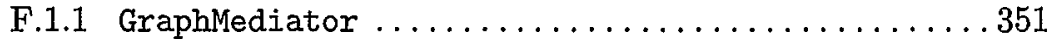

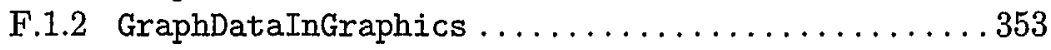

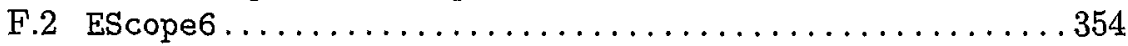

F.2.1 Reading Properties from GuiFacade . . . . . . . . . 354

F.2.2 GraphicsFacade ...................... 355

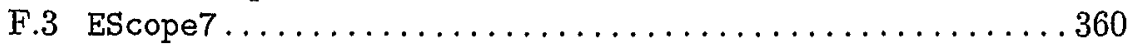

F.3.1 GraphUpdateEvent ............................ 360

F.3.2 The Scale Interfaces . . . . . . . . . . . . . . . 361

F.3.3 GraphicsFacade: Pop-up Menu and Associated

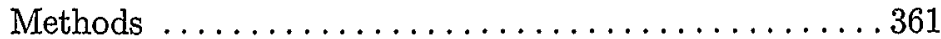

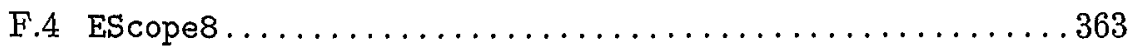

F.4.1 DataServerProxy: getPlotData .............. 363

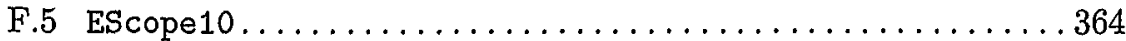

F.5.1 ServerSelectDialog. .......................... 364

F.5.2 ConnectAction Inner Class from GuiFacade . . . . . . . . 368

F.5.3 Factory Interface and Factory Classes . . . . . . . . . . 369

F.5.4 TextDataServer ........................ 370

F.6 EScope11........................................ 375

F.6.1 DataServerHandler ...................... 375

References ........................................ 379

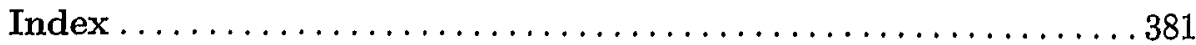

